\title{
Slot Antenna Design for a Wirelessly Powered Implantable Microcooler for Neuronal Applications
}

\author{
H. Dinis, J. Fernandes, P. M. Mendes \\ CMEMS, University of Minho, Guimarães, Portugal, hugodedinis@gmail.com
}

\begin{abstract}
Implantable medical devices are becoming smaller by the day, with more efficient electronics and smaller power demands. Nevertheless, there are some applications in which power demands are inherently high, and solutions must be found in order to keep the devices as small as possible. In this paper, we propose an antenna to be used in wirelessly powering a focal brain cooling implant based on a Peltier device. This antenna is designed in order to act as a heatsink for the device with the goal of minimizing its final volume, therefore design constrains such as size limitations and geometry restrictions are considered.
\end{abstract}

Index Terms-slot antenna, wireless power transfer, implant.

\section{INTRODUCTION}

Due to remarkable technological advances in the past decades, implantable electronic devices are becoming ever smaller and more efficient, which drives their suitability to many new applications to levels never seen before. The medical field is one of the most important ones, with examples of devices such as implantable chemical sensors [1], glucose and oxygen sensors for diabetics [2], neural implants $[3,4]$, cardiac pacemakers [5], and cochlear implants [6]. From the previous examples, it is possible to notice that there is an ongoing demand for implantable devices, which tend to become smaller and less power demanding [7].

An implantable device's size is most of the times limited by its battery size, a direct consequence of its power requirements. In order to minimize an implant's volume, its bulky batteries must be replaced by wireless powering modules, which can sometimes be coupled to smaller, rechargeable batteries [7].

When it comes to wirelessly powering an implanted device, electromagnetic radiation is adequate to transport energy over long distances and presents one of the highest miniaturization potentials and highest power density per unit area [8], but several challenges arise. RF signal attenuation in the biological lossy tissues, and reflections in the air-tissue and tissue-tissue interfaces negatively affect transmission efficiency. Also, power absorption by the tissues must be carefully controlled, as SAR limits must not be exceeded, in order to fulfil the patient safety rules.

We aim at the development of a wireless power transfer module for a small implantable device which will be used for focal brain cooling as an epilepsy controlling mechanism.
The design must bear in mind volume restrains, power needs of the implant and patient safety guidelines, more specifically SAR limits. In order to keep the final volume as small as possible, the device's heatsink, a metal sheet, will have a dual purpose, as it will also be acting as an antenna.

In the next section of this paper, a prototype of the implant is presented and its requirements and limitations are detailed and explained. In section III, the antenna development process is described, a prototype is tested and final considerations about the powering of the implant are presented.

\section{NEUROMOdULATION DEVICE}

The implantable neuromodulator under development is based on a Peltier device's capability to generate temperature gradients when powered with an electric current. A photograph of the current prototype is presented in Fig. 1. The actual device is obtained by the integration of CMOS electronics, a Peltier element, and a heatsink. From previous work, it was determined that the device requires $100 \mathrm{~mW}$ of power in order to achieve the desired level of brain cooling. Due to this high power requirement, and to avoid the use of large batteries that would increase the device size, a solution is to use a wireless powering module, which requires an antenna. The CMOS module would then be responsible for RF-DC conversion in order to power the Peltier.

Since miniaturizing the implant's volume is a key objective of our project, the addition of an antenna to this design has an impact on full device size. Currently, it has an area of $20 \times 20 \mathrm{~mm}^{2}$, mostly due to its heatsink, a key component that keeps the Peltier hot side temperature below acceptable limits, while the cold side reaches the desired temperatures. However, as the heatsink is a sheet of metal and the largest component of the prototype, we have decided to explore the possibility of using it as an antenna as well.

The device is being developed for testing in a rat's brain, therefore its dimensions dictate that, in order to have the

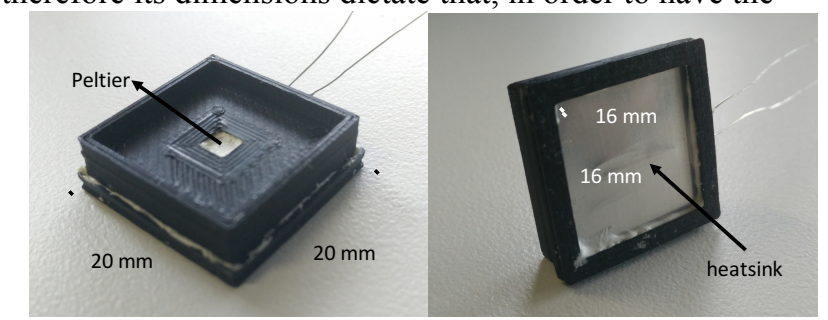

Fig 1. Implantable device's prototype, top (left) and bottom (right) views. 
Peltier's cold side in contact with brain, the heatsink/antenna would be left outside the animal's head, in contact with air. Consequently, antenna development is conducted bearing in mind that the antenna will operate in air, and not inside biological tissue.

\section{ANTENNA DEVELOPMENT}

When developing an antenna for an implantable device, design constrains such as volume and weight limitations are expected and must be worked around. In this particular case, and considering that the antenna will also serve as a planar heatsink, complex geometries are mostly impossible, due to the fact that they could severely cripple the device's heat conductivity by reducing its effective area. The chosen design was a slot antenna [9], which only requires a cut in the metal sheet. Still, the placement of the cut is of utmost importance. If it is done in middle of the heatsink, it will effectively reduce the heat spreading area to half, therefore it must be placed as close as possible to the edges of the metal sheet.

A single opening on the top of the metal sheet with varying width and length was initially considered, as illustrated in Fig 2, and these parameters were optimized for antenna gain. Length was kept below $16 \mathrm{~mm}$, due to size limitations of the metal sheet, while width was limited to 2 $\mathrm{mm}$ because larger slots would take up too much heatsink area. As the antenna is going to be used in air, the HFSS model consists of the slot antenna surrounded by an air box.

\section{A. Slot width optimization}

Slot width was simulated for values between $0.5 \mathrm{~mm}$ and $2 \mathrm{~mm}$, with a $0.5 \mathrm{~mm}$ step. The feed point location was studied through a parametric sweep. Return loss was analysed for each one and, for the cases where it was below $-10 \mathrm{~dB}$, the feed location that resulted in the highest gain was selected. The $S_{11}$ plot and radiation pattern results are shown in Fig. 3 and Fig. 4, respectively.

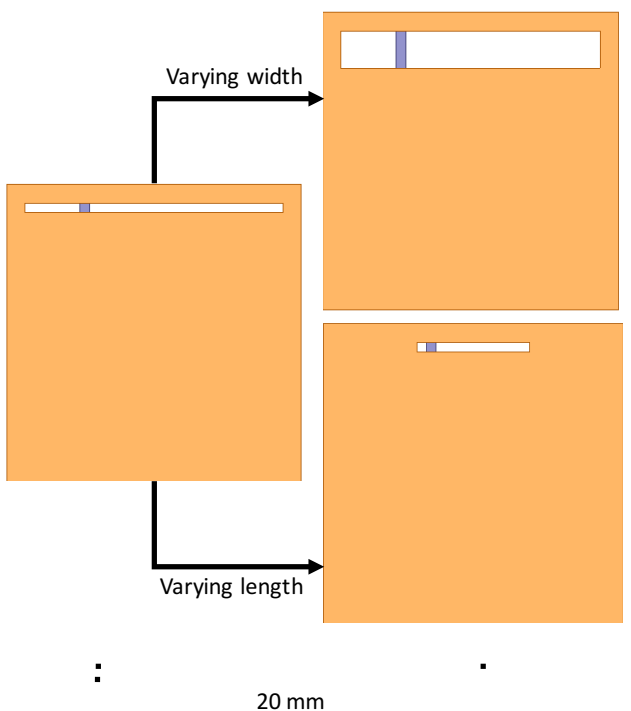

Fig. 2. Slot dimension parameters that were studied in this work.

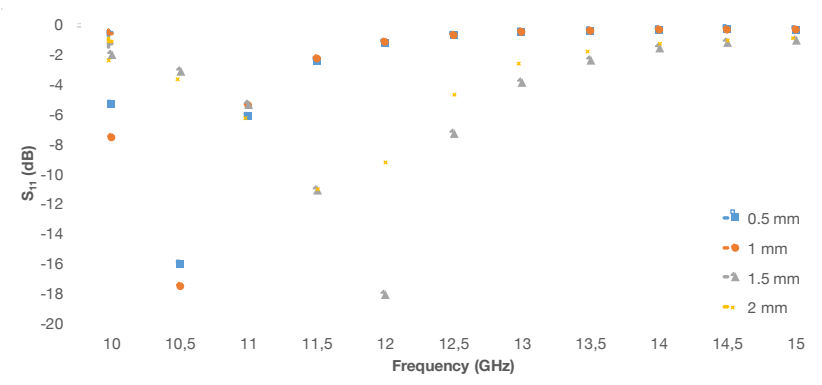

Fig. 3. Simulated $S_{11}$ parameter of the four different slot widths.
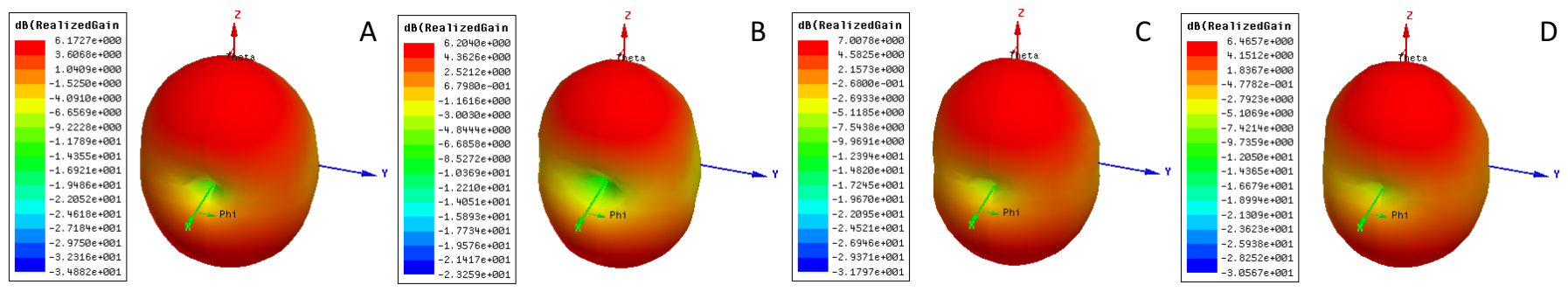

Fig. 4. Simulated radiation pattern of different slot widths at their resonant frequencies. (A) $0.5 \mathrm{~mm}$. (B) $1 \mathrm{~mm}$. (C) $1.5 \mathrm{~mm}$. (D) $2 \mathrm{~mm}$
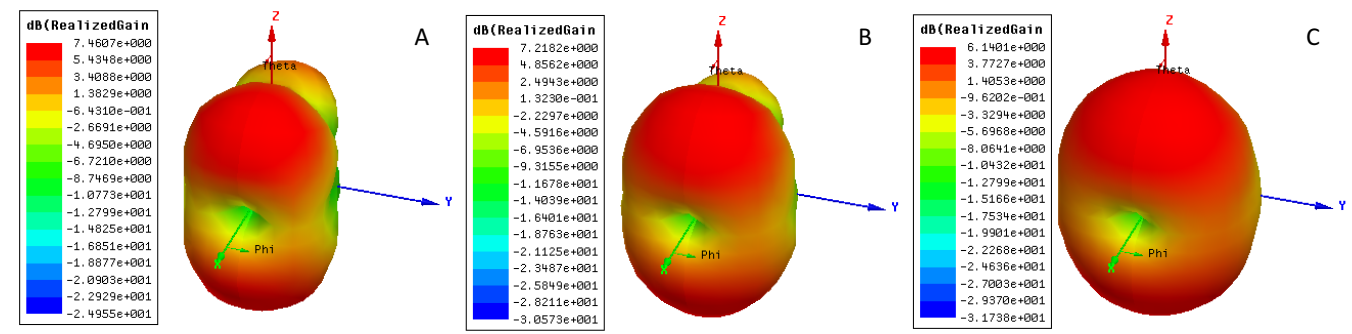

Fig. 5. Simulated radiation patter of different slot lengths at their resonant frequencies. (A) $10 \mathrm{~mm}$. (B) $12 \mathrm{~mm}$. (C) $14 \mathrm{~mm}$. 
For a width of $0.5 \mathrm{~mm}$ or $1 \mathrm{~mm}$, the operation frequency is the same, $10.5 \mathrm{GHz}$, but the latter has a slightly higher gain. The same happens with 1.5 and $2 \mathrm{~mm}$, at the frequency of $12 \mathrm{GHz}$, where the first presents a higher gain. When comparing widths of 1 and $1.5 \mathrm{~mm}$, we can't reach an immediate conclusion, because even though the second has a higher gain $(7.01 \mathrm{~dB}$ against $6.20 \mathrm{~dB})$, for a width of $1 \mathrm{~mm}$ the operation frequency is $1.5 \mathrm{GHz}$ lower, which can be beneficial in terms of tissue attenuation of the signal. Therefore, both values will be considered for a next optimization step, before any decision is made on which of the two will be used.

\section{B. Slot length optimization}

Slot length was simulated between 6 and $14 \mathrm{~mm}$, with a $2 \mathrm{~mm}$ step. Only lengths equal to or above $10 \mathrm{~mm}$ have shown resonant frequencies in the study's frequency range (2.5-15 GHz). Fig. 6 contains the $S_{11}$ plot of such cases, and Fig. 5 the respective radiation patterns. From the analysis of the results, it is possible to see that, for a slot length of $10 \mathrm{~mm}$, a $7.46 \mathrm{~dB}$ gain is obtainable at $15 \mathrm{GHz}$. With $12 \mathrm{~mm}, 7.22 \mathrm{~dB}$ at $13.5 \mathrm{GHz}$ are possible, and with $14 \mathrm{~mm}$, $6.14 \mathrm{~dB}$ at $10.5 \mathrm{GHz}$ are obtained. While for higher slot lengths lower operation frequencies are observed, the gain also decreases, which renders a choice based on these criteria alone impossible.

\section{U-shaped slot}

In the two previous subsections, we have studied the effect of the slot's width and length in the antenna's

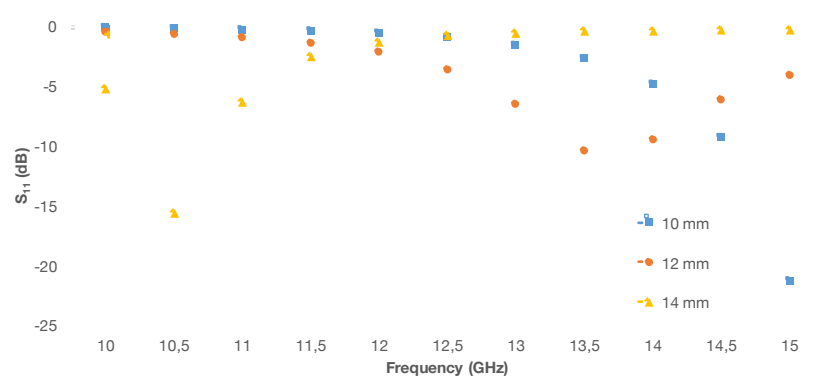

Fig. 6. Simulated $S_{11}$ parameter of antennas with 10,12 and $14 \mathrm{~mm}$ behavior. Even though it was possible to rule out some of the simulated parameters, some values of width and length still produced results that couldn't be immediately excluded or preferred over the others. More importantly, high operation frequencies have been obtained in all situations, which is unfavourable due to both powering setup limitations and higher SAR, as it is proportional to tissue conductivity, which in turn increases with frequency [10].

Therefore, a U-shaped slot, exemplified in Fig 7, was attempted in order to reduce antenna operation frequency. This design allows for a lengthier slot without sacrificing any significant heat sink area, as the central metal sheet remains intact. For the new design, the previously studied parameters that couldn't be immediately ruled out were chosen and combined, i.e., lengths of 10, 12 and $14 \mathrm{~mm}$ with widths of 1 and $1.5 \mathrm{~mm}$, which originated a total of 6 different slot designs to be simulated and considered.

Having simulated the six possible combinations of length and width, as mentioned above, the results were evaluated and, like it was previously done, the parameter combinations which yielded the worst results were removed from consideration. Fig 8 and Fig 9 contain the radiation patterns and $S_{11}$ parameters, respectively, of the designs with the most satisfying results.

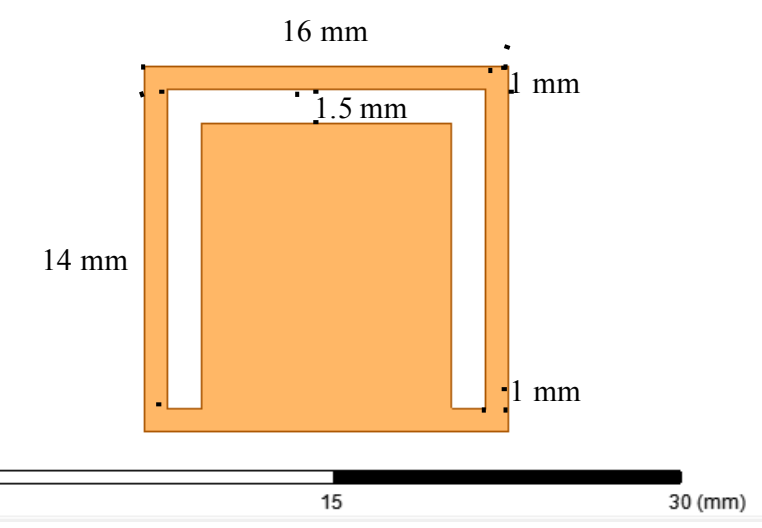

Fig. 7. HFSS model of a U-slot antenna/heatsink.

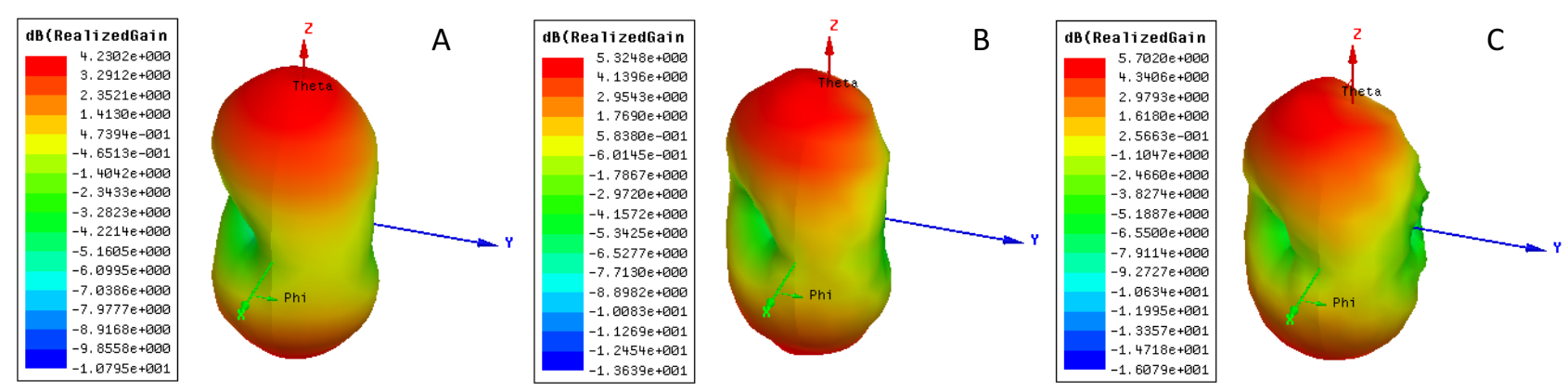

Fig. 8. Simulated radiation patterns of the three U-shaped slots. (A) Width $1.5 \mathrm{~mm}$ and length $14 \mathrm{~mm}$. (B) Width $1 \mathrm{~mm}$ and length $10 \mathrm{~mm}$. (C) Width $1.5 \mathrm{~mm}$ and length $10 \mathrm{~mm}$. 


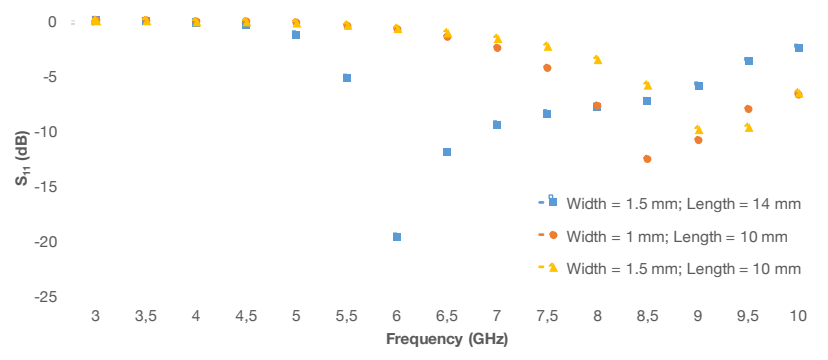

Fig. 9. Simulated S11 parameter of U-slot antennas with the chosen width and length values.

For a slot width of $1.5 \mathrm{~mm}$ and length of $14 \mathrm{~mm}$ (combination A), a gain of $4.23 \mathrm{~dB}$ at the operation frequency of $6 \mathrm{GHz}$ was obtained, which is the lowest frequency value, as expected, since this was the longest slot that was tested. As for the other two results, occurring for a slot with $10 \mathrm{~mm}$ of length and widths of 1 (combination B) and $1.5 \mathrm{~mm}$ (combination $\mathrm{C}$ ), it is visible that the operation frequencies are 8.5 and $9 \mathrm{GHz}$, and the gains 5.32 and $5.70 \mathrm{~dB}$, respectively.

Concerning these three results, a choice has to be made as to what combination of parameters will be prototyped and experimentally tested. Combination A, despite operating at the lowest frequency, requires the largest slot, and has the lowest gain. Combination B has a larger gain with a smaller slot, allowing further miniaturization of the device, which is why it is favoured over combination A. Combination $\mathrm{C}$, in its turn, has a similar gain to $\mathrm{B}$, but a slightly higher operation frequency and thicker slot, hence the final choice pending towards combination B.

\section{Fabricated slot antenna}

Having selected the slot design to be used, a first iteration of the antenna was fabricated with off-the-shelf materials, namely Rogers RO4003 substrate. The slot was carved from the substrate's top copper layer, while the bottom one was etched out. Holes for an SMA connector were drilled and it was soldered to the slot antenna, resulting in the device shown in Fig. 10.

The simulated and measured $\mathrm{S}_{11}$ parameter of the antenna are presented in Fig. 11 for a frequency range between 6.5-8.5 GHz, as well as the measured $\mathrm{S}_{21}$ between the fabricated antenna and a horn antenna for three distances.

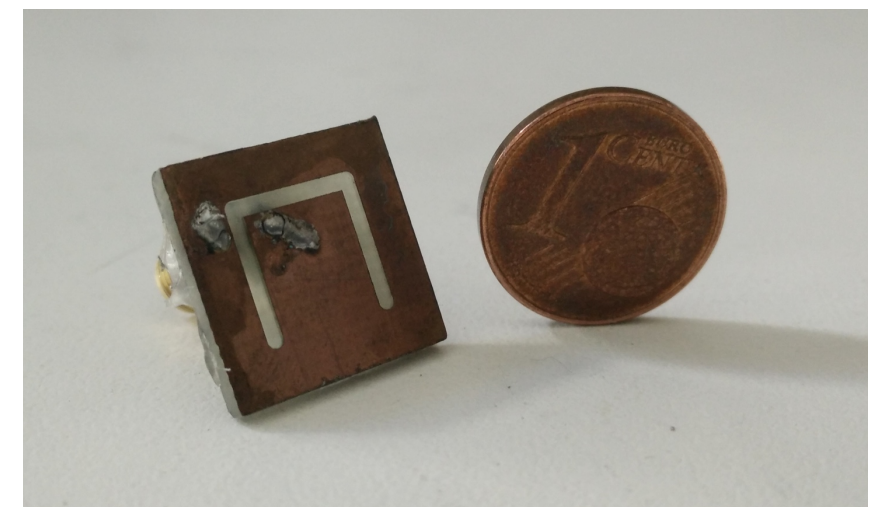

Fig. 10. Slot antenna prototype.

The $S_{11}$ results show great agreement with the simulation. The antenna operates through the entire frequency range, but has a prominent $\mathrm{S}_{11}$ minimum around $7.5 \mathrm{GHz}$, which coincides with a slightly higher $\mathrm{S}_{21}$ value of $-18.45 \mathrm{~dB}$ at 10 $\mathrm{cm}$. Additionally, since the $\mathrm{S}_{21}$ lines for the three distances present the same behaviour all over the studied frequency range, it is possible to affirm that no signal reflections are interfering with this measurement. The operation frequency was reduced from the previously simulated $8.5 \mathrm{GHz}$ to $7.5 \mathrm{GHz}$ due to the presence of the Roger's substrate dielectric material coupled to the original slot antenna.

Having determined that a $-18.45 \mathrm{~dB}$ link between the horn and target antennas was achieved, it was possible to calculate what the transmitted power must be in order to deliver $100 \mathrm{~mW}$ to the brain cooling device's antenna. Through simple calculations, it was determined that the power source must supply $7 \mathrm{~W}$ to the horn antenna.

Such a large power output may, however, be incompatible with in-vivo implantation due to SAR regulations. An HFSS model, consisting on the fabricated antenna placed $2 \mathrm{~mm}$ above brain tissue, was designed in order to determine how much power can be delivered to the slot antenna without surpassing the $2 \mathrm{~W} / \mathrm{kg}$ SAR limit. It was noted that due to the presence of the brain below the antenna, its operation frequency shifted to $7 \mathrm{GHz}$. At that frequency, the delivered power was determined to be $3.2 \mathrm{~mW}$, which is insufficient to power the implantable brain cooling device, and is an issue that must be resolved in future work. Further antenna optimization is required in order to account for the influence of brain matter in its proximity. 


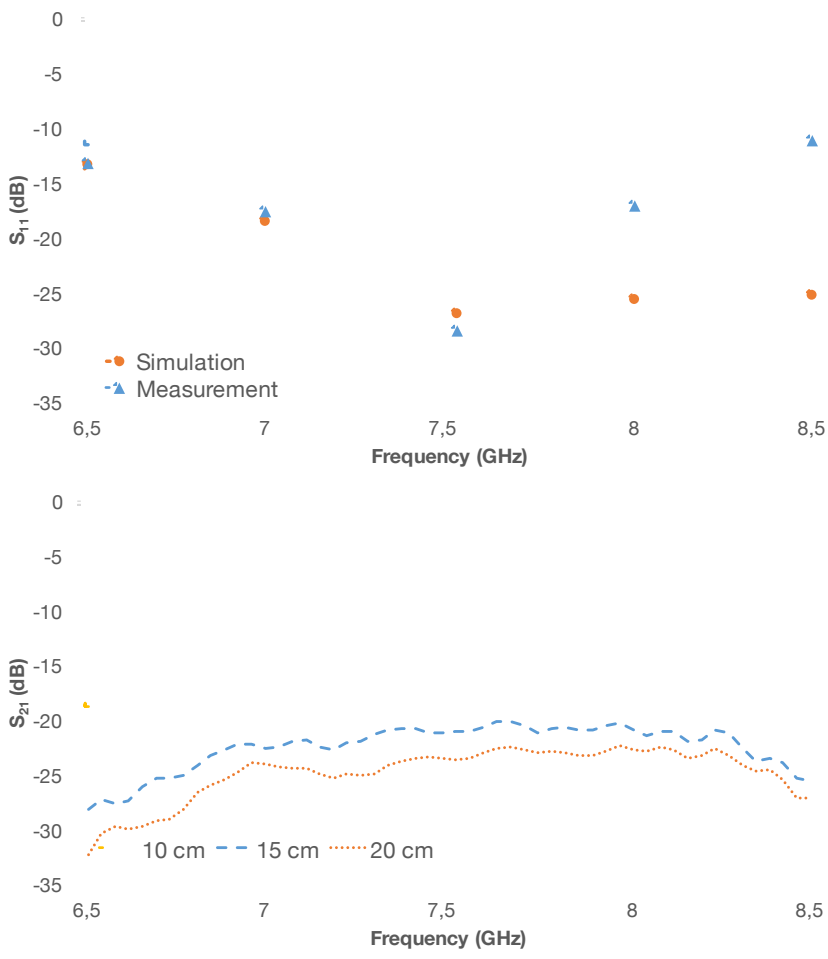

Fig. 11. S parameters of the U-shaped slot antenna (measured and simulated).

\section{CONCLUSIONS AND FutURE WORK}

This work focused on the first stage of the development of an antenna to wirelessly power an implantable focal brain cooling device. As the objective is an implantable device, volume minimization is a key requirement, therefore it was decided that the antenna was to be integrated with the device's heatsink, its largest component, comprised of a metal sheet. A slot cut into the sheet was enough to create an antenna that didn't significantly affect the heatsink's ability to achieve its heat spreading purpose. The final design, after several iterations, was a U-shaped slot with a width of $1 \mathrm{~mm}$ and length of $10 \mathrm{~mm}$ in each direction, with a total length of $30 \mathrm{~mm}$. From SAR studies, it was possible to estimate that, without surpassing the established SAR limit, only a small amount of power, $3.2 \mathrm{~mW}$, could be delivered to the implant, which is not enough for its desired operation. As future work on the antenna, further enhancement has to be performed, in order to increase its gain and lower its operation frequency, which will help to keep SAR values lower. Furthermore, antenna characterization when integrated with the implant has to be performed. The wireless link will be the main focus on future endeavours, and a multidimensional approach will be attempted, resorting to the possibility of using multiple transmitters, multiple receptors, pulsed waves and multipolarization, for example, in order to reduce SAR and, therefore, allow for more power transmission from the outside world to the implant.

\section{ACKNOWLEDGMENT}

This work is supported by FCT with the reference project PTDC/EEI-TEL/5250/2014, by FEDER funds through Projecto 3599 - Promover a Produção Científica e Desenvolvimento Tecnológico e a Constituição de Redes Temáticas (3599-PPCDT).

\section{REFERENCES}

[1] M. Frost and M. Meyerhoff, "Implantable chemical sensors for realtime clinical monitoring: progress and challenges," Curr. Opin. Chem. Biol., vol. 6, no. 5, pp. 633-641, 2002.

[2] B. McKean and D. Gough, "A telemetry-instrumentation system for chronically implanted glucose and oxygen sensors," IEEE Trans. Biomed. Eng., vol. BME-35, pp. 526-532, 1988.

[3] K. Wise, D. Anderson, J. Hetke, D. Kipke, and K. Najafi, "Wireless implantable microsystems: high-density electronic interfaces to the nervous system," Proc. IEEE, vol. 91, no. 1, pp. 76-97, 2004.

[4] R. H. Olsson and K. D. Wise, "A three-dimensional neural recording microsystem with implantable data compression circuitry," IEEE $J$. Solid-State Circuits, vol. 40, no. 12, pp. 2796-2804, 2005.

[5] M. Rasouli and L. Phee, "Energy sources and their development for application in medical devices," Expert Rev. Med. Devices, vol. 7, no. 5, pp. 693-709, 2010.

[6] K. Bazaka and M. Jacob, "Implantable Devices: Issues and Challenges", vol. 2, no. 1. 2012

[7] H. Dinis, "Performance Assessment of Electrically Small Antennas for Implantable Microsystems with Wireless Power and Communications", Universidade do Minho, 2015.

[8] E. Katz, "Implantable Bioelectronics". 2014.

[9] K. Wong, "Planar Antennas for Wireless Communications", New Jersey, USA: Wiley. 2003.

[10] S. Gabriel, R. W. Lau, and C. Gabriel, "The dielectric properties of biological tissues: II. Measurements in the frequency range $10 \mathrm{~Hz}$ to 20 GHz.," Phys. Med. Biol., vol. 41, no. 11, pp. 2251-2269, 1996. 